\title{
Severe Hypokalemia and Thyrotoxic Paralysis from Painless Thyroiditis Complicated by Life-threatening Polymorphic Ventricular Tachycardia and Rhabdomyolysis
}

\author{
Kyung Yoon Chang ${ }^{1}$, Su-Hyun Lee ${ }^{1}$, Hoon Suk Park ${ }^{1}$, Sun-Hee Ko ${ }^{2}$, \\ Yu-Bae Ahn ${ }^{2}$ and Hyung Wook Kim ${ }^{1}$
}

\begin{abstract}
A 61-year-old man presented with lower extremity paralysis and severe hypokalemia. His thyroid function test showed thyrotoxicosis. Despite attempts to correct his hypokalemia, he developed pulseless polymorphic ventricular tachycardia two hours later. He was successfully resuscitated after defibrillation. We performed continuous venovenous hemodiafiltration for 10 days due to acute kidney injury and rhabdomyolysis. We observed life-threatening polymorphic ventricular tachycardia requiring urgent defibrillation, as well as rhabdomyolysis requiring dialysis during the transient thyrotoxic phase of painless thyroiditis. Pay attention to the possibility of the development of life-threatening ventricular tachycardia associated with hypokalemia in the setting of thyroiditis and thyrotoxic paralysis.
\end{abstract}

Key words: hypokalemia, painless thyroiditis, polymorphic ventricular tachycardia, rhabdomyolysis, thyrotoxic paralysis

(Intern Med 53: 1805-1808, 2014)

(DOI: 10.2169/internalmedicine.53.2419)

\section{Introduction}

Graves' disease is the most common cause of thyrotoxic periodic paralysis (1), although it can occur due to any cause of hyperthyroidism. Only two patients with thyrotoxic periodic paralysis due to a painless thyroiditis have been reported $(2,3)$. There are a few descriptions of fatal ventricular arrhythmias in patients with thyrotoxicosis due to Graves' disease, but only one case with painless thyroiditis has been published (2). We herein describe a rare and lifethreatening case of painless thyroiditis that was complicated by severe hypokalemia, thyrotoxic paralysis, polymorphic ventricular tachycardia (VT), and rhabdomyolysis-induced acute kidney injury that required dialysis.

\section{Case Report}

A 61-year-old man presented with lower extremity paraly- sis without sensory loss. His blood pressure was 141/93 $\mathrm{mmHg}$, and his pulse was 118 beats/min. He had not taken any drugs, including diuretics. There was no family history of thyroid disease or periodic paralysis. He did not have diarrhea, vomiting, or symptoms of thyrotoxicosis, such as goiter, weight loss, palpitations, tremors, or proptosis. Palpation of the thyroid did not reveal either tenderness or nodules.

Initial laboratory analysis showed the following: sodium, $142.9 \mathrm{mEq} / \mathrm{L}$; potassium, $1.9 \mathrm{mEq} / \mathrm{L}$; chloride, $107.7 \mathrm{mEq} /$ $\mathrm{L}$; calcium, $9.5 \mathrm{mg} / \mathrm{dL}$; phosphorus, $2.8 \mathrm{mg} / \mathrm{dL}$; magnesium, $2.3 \mathrm{mg} / \mathrm{dL}$; creatine phosphokinase (CPK), $176 \mathrm{U} / \mathrm{L}$ (normal range 60-190); CPK-MB, $1.44 \mathrm{ng} / \mathrm{mL}$ (normal range 0.16.7); lactate dehydrogenase (LDH), $217 \mathrm{U} / \mathrm{L}$ (normal range 106-230); blood urea nitrogen (BUN), $17.8 \mathrm{mg} / \mathrm{dL}$; and creatinine, $0.9 \mathrm{mg} / \mathrm{dL}$. His spot urine potassium was $7 \mathrm{mEq} /$ $\mathrm{L}$, and his transtubular potassium gradient (TTKG) was 2.1\%. Blood gas analysis revealed $\mathrm{pH}, 7.402 ; \mathrm{pCO}_{2}, 34.4$ $\mathrm{mmHg} ; \mathrm{pO}_{2}, 73.7 \mathrm{mmHg} ; \mathrm{HCO}_{3}, 21.6 \mathrm{mmol} / \mathrm{L}$; and $\mathrm{SpO}_{2}$,

${ }^{1}$ Division of Nephrology, Department of Internal Medicine, St. Vincent's Hospital, The Catholic University of Korea, Korea and ${ }^{2}$ Division of Endocrinology, Department of Internal Medicine, St. Vincent's Hospital, The Catholic University of Korea, Korea

Received for publication December 31, 2013; Accepted for publication February 6, 2014

Correspondence to Dr. Hyung Wook Kim, khw@catholic.ac.kr 


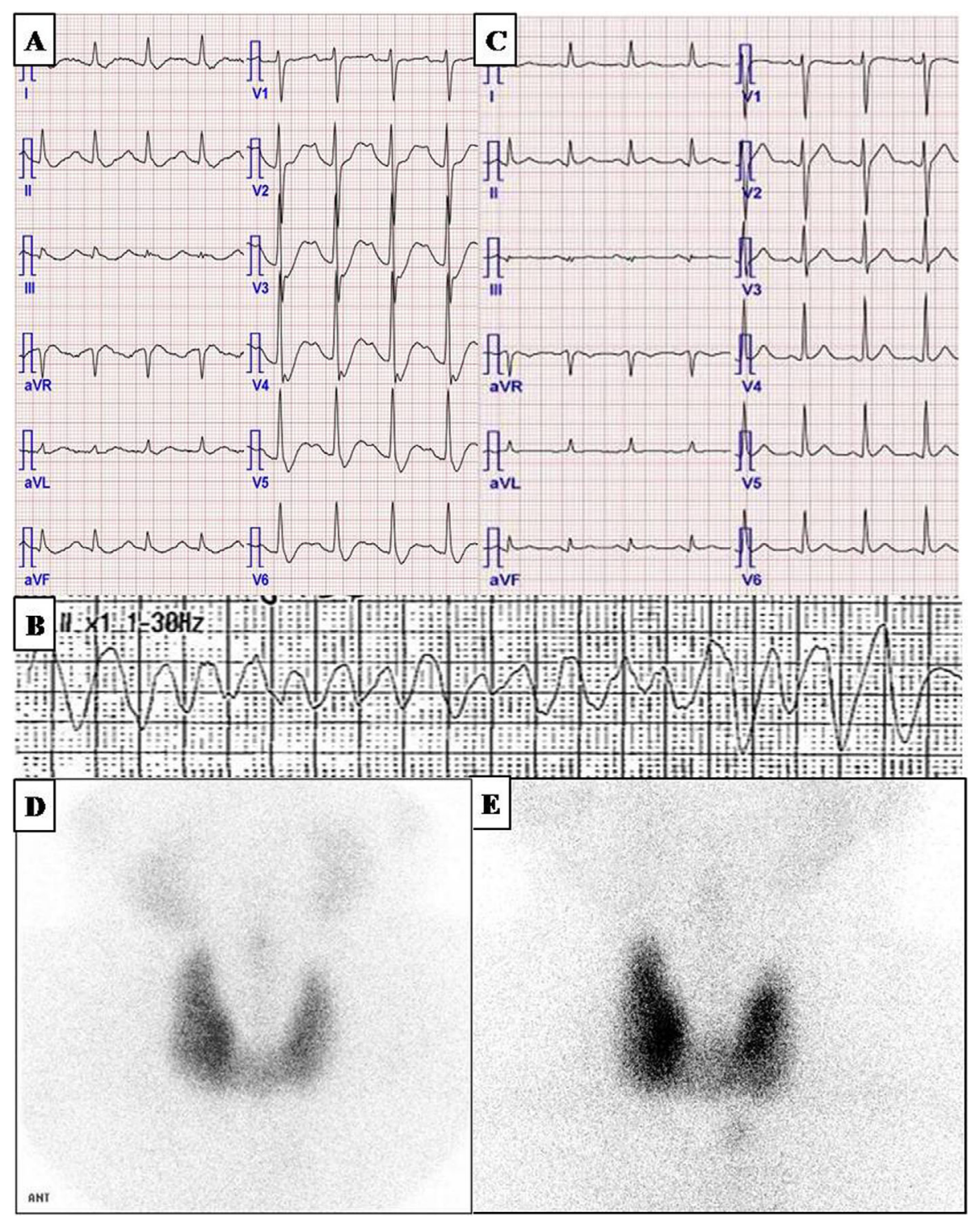

Figure 1. Changes in the ECG findings and thyroid scintigraphy after admission. The ECG findings on admission showed sinus tachycardia with a decrease in the amplitude of the $T$ wave and a prolonged QT interval (corrected QT interval=456 msec) (A). Two hours after admission, the patient developed polymorphic ventricular tachycardia (B). The patient was successfully resuscitated after two rounds of CPR and 23 rounds of defibrillation, and his ECG showed normal sinus rhythm with a normal QT interval (corrected QT interval=413 msec) (C). Two weeks after admission, thyroid scintigraphy with Tc-99m pertechnetate revealed a mildly, diffusely enlarged thyroid gland with nonhomogenous thyroid uptake, suggesting thyroiditis (D). Repeat thyroid scintigraphy after six months revealed normal uptake in the thyroid area $(E)$.

95.1\%. The patient's brain magnetic resonance imaging (MRI) and electroencephalography findings were normal. His thyroid function tests showed the following levels: thyroid-stimulating hormone (TSH), $0.02 \mu \mathrm{IU} / \mathrm{mL}$ (normal range 0.35-4.94); free thyroxine (FT4), $1.70 \mathrm{ng} / \mathrm{dL}$ (normal range 0.7-1.48); and triiodothyronine (T3), $204 \mathrm{ng} / \mathrm{dL}$ (normal range 58-159). These results were suggestive of thyrotoxicosis. The patient's levels of the TSH receptor antibody, anti-microsomal antibody, and anti-thyroglobulin antibody were $1.08 \mathrm{IU} / \mathrm{L}$ (normal range $<1.75$ ), $<10 \mathrm{U} / \mathrm{mL}$ (normal range $<30$ ), and $20 \mathrm{U} / \mathrm{mL}$ (normal range $<40$ ), respectively. We suggest painless thyroiditis as the cause of the patient's thyrotoxicosis. We hypothesized that the hypokalemia was the result of potassium redistribution into the cells secondary to thyroiditis, due to the patient's low TTKG and the lack of evidence of gastrointestinal and urinary potassium loss. The patient was treated with oral and intravenous potassium chloride, continuous electrocardiograph (ECG) monitoring, and regular serum potassium level checks. Anti-thyroid drugs were not administered.

The initial ECG showed sinus tachycardia with depression of the ST segment, a decrease in the amplitude of the $\mathrm{T}$ wave, and a prolonged QT interval (corrected QT interval = $456 \mathrm{~ms}$, normal range <440) (Fig. 1A). Two hours later, the 


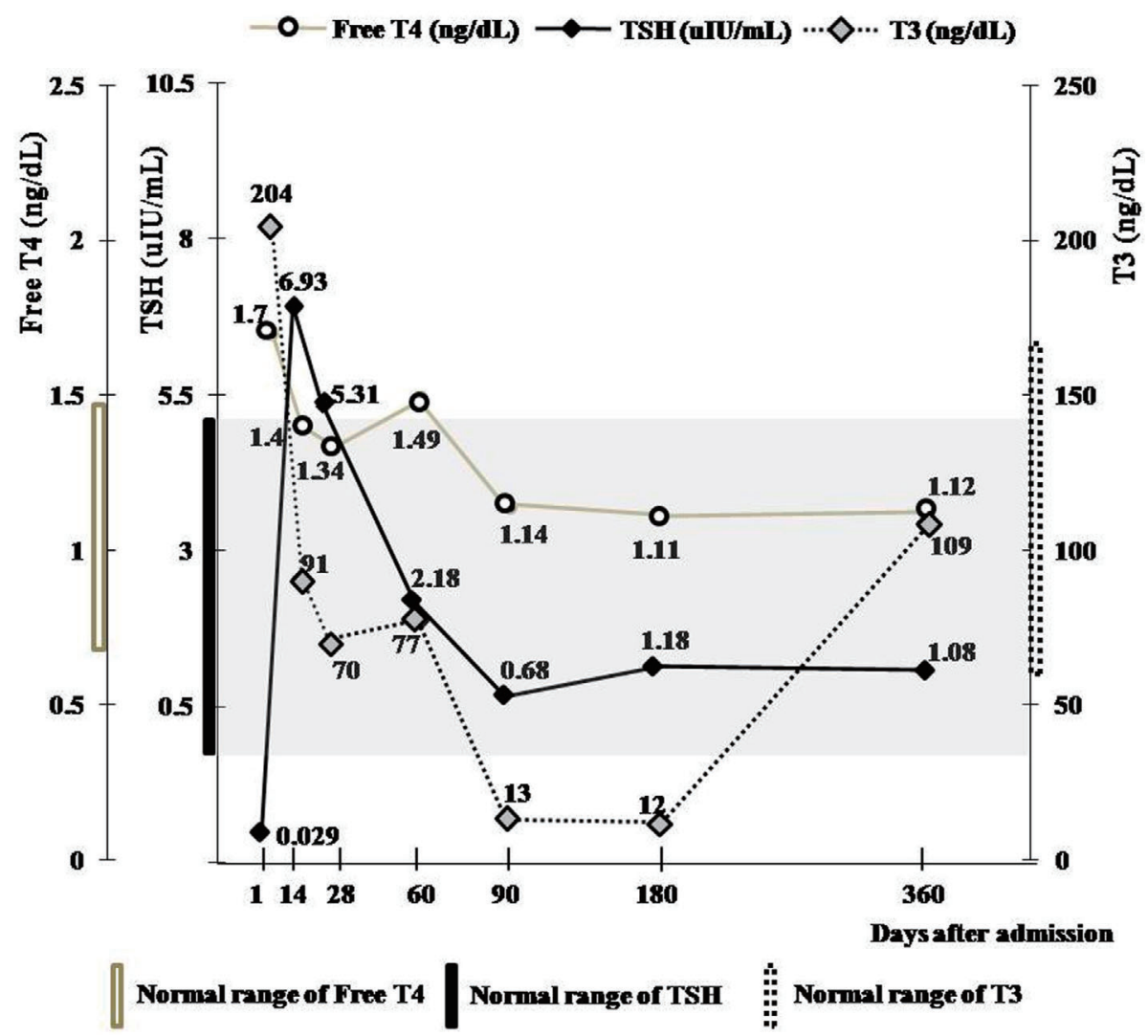

Figure 2. Changes in thyroid hormones after admission. Note: Normal range of TSH, 0.35-4.94 $\mu \mathrm{IU} / \mathrm{mL}$; normal range of FT4, 0.7-1.48 ng/dL; normal range of T3, 58-159 ng/dL. TSH: thyroidstimulating hormone, FT4: free thyroxine, T3: triiodothyronine

patient lost consciousness, and pulseless polymorphic VT began (Fig. 1B). We injected intravenous magnesium sulfate. The patient was successfully resuscitated after receiving two rounds of cardiopulmonary resuscitation (CPR) and 23 rounds of direct current defibrillations at $200 \mathrm{~J}$ of biphasic energy for 100 minutes. After serum potassium levels were corrected, the ECG showed normal sinus rhythm with a normal QT interval (corrected QT interval $=413 \mathrm{msec}$ ) (Fig. 1C). An echocardiogram showed normal cardiac function without evidence of structural heart disease.

After resuscitation, the patient developed acute kidney injury and severe rhabdomyolysis. On day 7 of admission, laboratory data showed BUN, $114.6 \mathrm{mg} / \mathrm{dL}$; creatinine, 7.8 $\mathrm{mg} / \mathrm{dL}$; LDH, 12,090 U/L; CPK, >62,000 U/L; CPK-MB/ $\mathrm{CPK}$ ratio, $<6 \%$; and decreased urine volume. We performed continuous venovenous hemodiafiltration for 10 days, followed by intermittent hemodialysis for 18 days. After renal replacement therapy for 28 days, the patient's renal function recovered, and hemodialysis was stopped.

At two weeks, the patient's TSH, FT4, and T3 values were $6.93 \mu \mathrm{IU} / \mathrm{mL}, 1.4 \mathrm{ng} / \mathrm{dL}$, and $91 \mathrm{ng} / \mathrm{dL}$, respectively, thus suggesting subclinical hypothyroidism. We performed thyroid scintigraphy with Tc-99m pertechnetate, which revealed a mildly and diffusely enlarged thyroid gland with nonhomogenous uptake, thus indicating thyroiditis (Fig. 1D). At the three-month follow-up, the TSH, FT4, and T3 values were $0.68 \mu \mathrm{IU} / \mathrm{mL}, 1.14 \mathrm{ng} / \mathrm{dL}$, and $130 \mathrm{ng} / \mathrm{dL}$, respectively, suggesting euthyroidism. Six months after admission, thyroid function tests were normal (Fig. 2), and thyroid scintigraphy with Tc-99m pertechnetate showed normal uptake in the thyroid area (Fig. 1E). The patient's renal function improved slowly but has not completely recovered; his BUN and creatinine levels were $23 \mathrm{mg} / \mathrm{dL}$ and $1.8 \mathrm{mg} /$ $\mathrm{dL}$, respectively, at six months after admission.

\section{Discussion}

Painless thyroiditis is characterized by a brief phase of thyrotoxicosis lasting two to four weeks, subsequent hypothyroidism for one to three months, and then resolution. This is considered a variant of chronic autoimmune thyroiditis, indicating that the patient is on the thyroid autoimmune disease spectrum (4). The diagnosis of painless thyroiditis is based upon clinical manifestations and laboratory findings. In patients with hyperthyroidism and without clinical manifestations of Graves' disease, a radioiodine uptake test is necessary to differentiate painless thyroiditis from Graves' disease. In contrast to Graves' disease, thyroid radioiodine uptake is very low during the thyrotoxic phase of painless thyroiditis. Many patients with painless thyroiditis do not require treatment during either the hyperthyroid or the hypothyroid phases because thyroid dysfunction is transient and rarely severe.

Hypokalemia during the transient thyrotoxic phase of 
painless thyroiditis can cause thyrotoxic paralysis and serious polymorphic VT. There have been a few reports of fatal ventricular arrhythmias associated with hypokalemia and thyrotoxicosis due to Graves' disease $(5,6)$, but only one case of painless thyroiditis has been described (2).

Polymorphic VT is a rapid and hemodynamically unstable rhythm, and urgent defibrillation is usually necessary. It may persist and can degenerate into ventricular fibrillation, which will lead to sudden death in the absence of prompt treatment. Polymorphic VT that occurs in the setting of QT prolongation is a distinct arrhythmia called torsade de pointes. In the absence of QT prolongation, polymorphic VT is most often due to ischemia, which can be overt or silent (7). Other causes of polymorphic VT are drug therapy, hypokalemia, hypomagnesemia, and congenital long QT syndrome (8). The management of polymorphic VT is immediate defibrillation if the patient is hemodynamically unstable (9). Further therapy is required to treat underlying disorders and prevent recurrences.

Severe hypokalemia can induce ventricular arrhythmias. In the Purkinje fibers of the cardiac conducting system, hypokalemia causes depolarization and leads to increased membrane excitability and arrhythmias. Hypokalemia delays ventricular repolarization by inhibiting potassium channel activity (10) and can also cause rhabdomyolysis. Potassium release from muscle cells normally mediates vasodilation and an appropriate increase in muscle blood flow. However, decreased potassium release from muscle cells due to hypokalemia can decrease the blood flow to muscles, leading to ischemic rhabdomyolysis (11). In the present case, severe hypokalemia could have been the cause of rhabdomyolysis; however there is a good possibility that it was the result of resuscitation and defibrillations, which can also increase CPK and LDH. The release of CPK after elective cardioversion correlates with the cumulative energy delivered, thus indicating increased skeletal muscle damage with greater energy (12-14). Prolonged resuscitative efforts involving repeated defibrillations may predispose patients to rhabdomyolysis.

In this case, severe hypokalemia during the transient thyrotoxic phase of painless thyroiditis caused life-threatening polymorphic VT requiring urgent defibrillation, rhabdomyolysis requiring dialysis, and thyrotoxic paralysis. The patient's renal function after acute kidney injury has not completely recovered. Physicians should therefore pay attention to the possibility of the development of life-threatening VT associated with hypokalemia in the setting of thyroiditis and thyrotoxic paralysis.

The authors state that they have no Conflict of Interest (COI).

\section{References}

1. Lin SH. Thyrotoxic periodic paralysis. Mayo Clin Proc 80: 99$105,2005$.

2. Lee JI, Sohn TS, Son HS, et al. Thyrotoxic periodic paralysis presenting as polymorphic ventricular tachycardia induced by painless thyroiditis. Thyroid 19: 1433-1434, 2009.

3. Oh SB, Ahn J, Oh MY, et al. Thyrotoxic periodic paralysis associated with transient thyrotoxicosis due to painless thyroiditis. J Korean Med Sci 27: 822-826, 2012.

4. Pearce EN, Farwell AP, Braverman LE. Thyroiditis. N Engl J Med 348: 2646-2655, 2003.

5. Ando T, Henmi T, Haruta D, et al. Graves' disease complicated by ventricular fibrillation in three men who were smokers. Thyroid 21: 1021-1025, 2011.

6. Nadkarni PJ, Sharma M, Zinsmeister B, Wartofsky L, Burman KD. Thyrotoxicosis-induced ventricular arrhythmias. Thyroid 18: 1111-1114, 2008.

7. Wolfe CL, Nibley C, Bhandari A, Chatterjee K, Scheinman M. Polymorphous ventricular tachycardia associated with acute myocardial infarction. Circulation 84: 1543-1551, 1991.

8. Roden DM. Drug-induced prolongation of the QT interval. N Engl J Med 350: 1013-1022, 2004.

9. Zipes DP, Camm AJ, Borggrefe M, et al. ACC/AHA/ESC 2006 guidelines for management of patients with ventricular arrhythmias and the prevention of sudden cardiac death: a report of the American College of Cardiology/American Heart Association Task Force and the European Society of Cardiology Committee for Practice Guidelines (Writing Committee to Develop Guidelines for Management of Patients With Ventricular Arrhythmias and the Prevention of Sudden Cardiac Death). J Am Coll Cardiol 48: 247346, 2006.

10. Guo J, Massaeli $\mathrm{H}, \mathrm{Xu}$ J, et al. Extracellular $\mathrm{K}^{+}$concentration controls cell surface density of $\mathrm{I}_{\mathrm{Kr}}$ in rabbit hearts and of the HERG channel in human cell lines. J Clin Invest 119: 2745-2757, 2009.

11. Knochel JP, Schlein EM. On the mechanism of rhabdomyolysis in potassium depletion. J Clin Invest 51: 1750-1758, 1972.

12. O'Neill PG, Faitelson L, Taylor A, Puleo P, Roberts R, Pacifico A. Time course of creatine kinase release after termination of sustained ventricular dysrhythmias. Am Heart J 122: 709-714, 1991.

13. Jakobsson J, Odmansson I, Nordlander R. Enzyme release after elective cardioversion. Eur Heart J 11: 749-752, 1990.

14. Minor RL Jr, Chandran PK, Williams CL. Rhabdomyolysis and myoglobinuric renal failure following cardioversion and CPR for acute MI. Chest 97: 485-486, 1990.

(C) 2014 The Japanese Society of Internal Medicine

http://www.naika.or.jp/imonline/index.html 